\title{
Régulation du secteur laitier en Algérie entre sécurité alimentaire et développement d'une production locale : synthèse
}

\author{
Fateh Mamine ${ }^{1 *}$ M'hand Fares ${ }^{1}$ Guillaume Duteurtre ${ }^{2}$ \\ Toufik Madani ${ }^{3}$
}

\begin{abstract}
Mots-clés
Lait, lait déshydraté, politique agricole, sécurité alimentaire, importation, Algérie
\end{abstract}

Submitted: 19 June 2020

Accepted: 9 February 2021

Published: 30 June 2021

DOI: $10.19182 /$ remvt.36362

\section{Résumé}

La sécurité alimentaire est au cœur des débats actuels concernant les politiques de développement agricole. Il s'agit notamment d'arbitrer entre le soutien à la production locale et l'ouverture au commerce international pour répondre à l'essor rapide de la demande. Cette question se pose de manière originale dans le secteur laitier algérien, où l'intervention de l'Etat concerne l'ensemble des maillons de la filière de la production à la consommation. Or, en dépit de ces mesures particulièrement coûteuses, la progression de la collecte de lait local reste limitée. L'Etat intervient aussi par des mécanismes de prix administrés et de quotas, qui favorisent l'importation de la poudre de lait afin d'assurer l'offre de produits laitiers à bas prix. Ce compromis aboutit au maintien de la dépendance du pays vis-à-vis des marchés internationaux et questionne sa capacité à élaborer une véritable stratégie de souveraineté alimentaire.

- Comment citer cet article : Mamine F., Fares M., Duteurtre G., Madani T., 2021. Regulation of the dairy sector in Algeria between food security and development of local production: Review. Rev. Elev. Med. Vet Pays Trop., 74 (2): 73-81, doi: 10.19182/remvt.36362

\section{INTRODUCTION}

Dans les pays développés, comme dans les pays en développement, l'intervention de l'Etat est un élément déterminant dans le fonctionnement des filières agroalimentaires (Giner, 2010 ; Kroll et Pouch, 2012). En Afrique, notamment, malgré les plans d'ajustement structurel et la libéralisation des économies intervenues depuis les années 1990 et 2000 , le rôle régulateur de l'Etat n'a pas disparu, surtout dans le secteur agroalimentaire où l'enjeu de la sécurité alimentaire est crucial. Cette dernière s'exerce désormais dans le cadre contraint des accords commerciaux internationaux (Laroche-Dupraz et Postolle, 2010).

Les récentes crises alimentaires au niveau mondial ont mis en lumière l'importance de la régulation publique. L'arrivée des acteurs privés dans l'arène économique comme conséquence directe de l'ajustement structurel a cependant transformé les modalités de cette intervention étatique (OCDE, 2005). Désormais, le rôle de l'Etat s'oriente

1. UMR Selmet, INRAE, 2 place Pierre Viala, 34000 Montpellier, France. 2. CIRAD, UMR SELMET, F-34398 Montpellier, France ; SELMET, Univ Montpellier, CIRAD, INRAE, Institut Agro, Montpellier, France.

3. Département d'agronomie, Université de Sétif, El Bez, Sétif 19000, Algérie. prioritairement vers la mise en place de mécanismes incitatifs publics et un encadrement institutionnel qui peut garantir l'arbitrage et le contrôle du marché (Janin et de Suremain, 2005). Toutefois, la crise sanitaire Covid-19 remet en cause tous les paradigmes de gestion des filières agroalimentaires en temps de crise puisque, contrairement à la crise alimentaire 2007-2008 liée à la pénurie alimentaire, les décideurs publics sont confrontés aux incertitudes liées à l'offre et à la demande, mais aussi à la capacité des opérateurs économiques de maintenir les chaînes d'approvisionnement (FAO, 2020).

L'Algérie a depuis longtemps recours à l'importation pour assurer l'approvisionnement du marché local en produits de première nécessité (lait, céréales, sucre, huiles). La dépendance alimentaire et la facture qui en découle se sont accrues au lendemain de l'Indépendance et le phénomène a continué de s'accentuer pendant les années 1990 et 2000 du fait de la croissance démographique rapide et du développement insuffisant de la production locale (Sari, 2001 ; Cherfaoui, 2003). Ainsi, entre 1962 et 2013, la consommation de lait est passée de 42 à $142 \mathrm{~kg}$ équivalents lait (EL) per capita et par an, tandis que la population passait de 11 à 38 millions d'habitants. En total cumulé, la consommation de lait a bondi de 0,5 à 5,5 millions de tonnes EL pendant cette période et la part des importations dans ce disponible laitier est restée constante à $44 \%$, représentant un saut de 0,22 à 2,5 millions de tonnes EL (http://faostat.fao.org). 
Aujourd'hui l'Algérie est le septième pays importateur de produits laitiers dans le monde en valeur ; les importations laitières algériennes représentent $24 \%$ des importations laitières africaines en valeur (Chatellier, 2019). En 2019, le secteur des produits laitiers a contribué pour $15,4 \%$ des importations alimentaires totales du pays en valeur, juste derrière les céréales qui comptaient pour 33,5 \% (DEP, 2020). Or, la flambée des prix des produits agricoles et agroalimentaires en 2007, année caractérisée par la hausse des prix des produits laitiers sur le marché mondial, a changé la donne en conduisant à une amplification de la facture d'importation (Madelin et al., 2007) qui s'est ainsi élevée à 1290 millions de dollars en 2008 (www.douane.gov. dz). Bien que l'Algérie soit dotée d'une rente pétrolière permettant de financer les dépenses d'importation, l'instabilité des prix du pétrole sur le marché international et l'horizon limité de sa rente mettent en péril la stabilité socioéconomique à long terme du pays. Pour ces raisons l'Etat algérien a revisité sa politique de structuration et de soutien d'une production locale de lait.

Dix ans après la mise en place du nouveau mode de régulation inscrit dans le cadre du programme du Renouveau agricole et rural (RAR) de 2009, il est possible d'en tirer un certain nombre d'enseignements. L'objectif de cet article était d'analyser les modalités que prend cette régulation publique du secteur laitier en Algérie ainsi que ses limites. Dans la première section, les concepts et les méthodes relatifs aux politiques laitières ont été présentés. Dans la deuxième section, la chronologie d'évolution des politiques de régulation de la filière lait en Algérie a été analysée. La troisième section a analysé les impacts de ces politiques dans les différents maillons de la filière avant de conclure.

\section{- CONCEPTS ET METHODE}

\section{Politiques de régulation laitières}

L'analyse des politiques de régulation du secteur agricole et agroalimentaire donne lieu à deux types d'approches économiques. Certains travaux cherchent à quantifier les implications économiques des différents types de mesures envisagées (mesure de prix, de subvention, ou d'innovation technique), en repérant notamment leurs coûts pour l'Etat, et les gains pour les différentes catégories d'acteurs. Il s'agit d'arbitrer notamment entre les gains (ou surplus) engendrés par telle ou telle mesure, et de fixer des niveaux de taxes. Ces travaux se basent notamment sur des modèles de marché qui permettent d'évaluer l'impact de ces mesures (Bouamra-Mechemache et al., 2002 ; Ledjou, 2012).

D'autres travaux cherchent à comprendre les conditions d'élaboration des différents types de mesures politiques, et discutent de leurs impacts sur différents indicateurs d'efficacité. Il ne s'agit alors pas de rendre compte uniquement de coûts et de gains économiques, mais de souligner dans leur diversité les impacts des différentes mesures négociées. Ces travaux peuvent alors évaluer les conséquences de ces mesures vis-à-vis de leurs objectifs concernant les quantités produites et transformées, les types de produits mis sur le marché, l'emploi rural, la pollution ou le coût en ressources naturelles par exemple. Pour évaluer ces politiques, il apparaît à la fois nécessaire de différencier les types de mesures envisagées (politiques de prix, de crédit, commerciales, sanitaires, technologiques, etc.), mais aussi la manière dont elles émergent dans l'agenda politique en fonction de l'évolution de la situation du secteur (Duteurtre et al., 2015 ; Gouin et Kroll, 2018 ; Ngom et al., 2019). C'est ce deuxième type d'approche qui a été privilégié dans ce travail afin de rendre compte de la pluralité des objectifs poursuivis par les politiques laitières algériennes.

Dans ce cadre, plusieurs traits fondamentaux des politiques de régulation du secteur laitier doivent être soulignés. En premier lieu, il faut considérer que cette régulation s'inscrit dans un contexte de grande instabilité des prix internationaux. L'indice des prix des produits laitiers publié par la FAO illustre cette grande instabilité qui concerne à la fois une volatilité dans le temps et des variations des prix relatifs entre produits (figure 1). C'est pour cela que les débats sur les niveaux de protection des marchés vis-à-vis du commerce international ont été particulièrement vifs ces dernières années (Duteurtre, 2009 ; Diarra et al., 2013 ; Baritaux et al., 2018). Ils s'insèrent dans les mutations politiques globales qui ont conduit à la libéralisation des politiques agricoles et des politiques commerciales de ces vingt dernières années. Plus spécifiquement, les politiques laitières des pays d'Afrique du Nord, comme celles des pays d'Afrique de l'Ouest, sont marquées par leur positionnement vis-à-vis des exportations de l'Union européenne qui constitue leur principal partenaire commercial. Or, depuis la suppression des quotas laitiers européens en 2015, les exportations laitières de l'Europe ont connu un regain d'agressivité commerciale qui a généré des tensions sur ces marchés (Duteurtre et Corniaux, 2019).

Un deuxième élément central relatif aux politiques de régulation du secteur laitier a trait à l'institutionnalisation des mécanismes de protection ou de gestion collective de l'offre. En effet, les filières laitières sont marquées par une pluralité d'acteurs impliqués et de produits qui influent sur les modes de gouvernance à mettre en œuvre (Duteurtre, 2008 ; Nguyen et al., 2017). Cette nature plurielle du secteur laitier génère une certaine complexité des mesures à adopter, qui conduisent de nombreux pays à mettre en place des offices (ou bureaux) du lait (milk boards). Si l'organisation et les rôles de ces offices sont variables, ils ont tous pour vocation de permettre la négociation, l'adaptation locale et la mise en œuvre des mesures de régulation sectorielle (Duteurtre, 2008 ; Gouin et Kroll, 2018). L'histoire et la diversité de ces institutions de régulation sectorielle rendent compte des spécificités nationales en matière de politiques laitières.

Enfin, il convient de reconnaître une certaine dualité des politiques laitières qui apparaissent comme le résultat de deux espaces de négociation, de justification et de mise en œuvre différents. D'un côté, les politiques d'appui à la production, qui sont principalement l'apanage des ministères en charge de l'agriculture et de l'élevage, rendent compte des arbitrages de l'Etat vis-à-vis de la production laitière locale. C'est au niveau de ces politiques que se construisent les choix publics pour soutenir ou redistribuer les revenus des producteurs et des industriels en fonction des prix du lait. D'un autre côté, les politiques commerciales, qui sont la plupart du temps élaborées par les services du ministère du commerce, rendent compte des choix du pays en matière d'ouverture ou de protection vis-à-vis des marchés internationaux pour gérer notamment les prix au consommateur (Corniaux et al., 2011 ; Ngom et al., 2019). Ces deux piliers des politiques de régulation laitière peuvent être plus ou moins bien intégrés selon l'importance du secteur laitier dans l'agenda politique (Corniaux et al., 2011). Lorsqu'ils existent, les bureaux ou offices interprofessionnels du lait rendent compte des tensions entre ces deux dynamiques d'élaboration des politiques et des rapports de force associés.

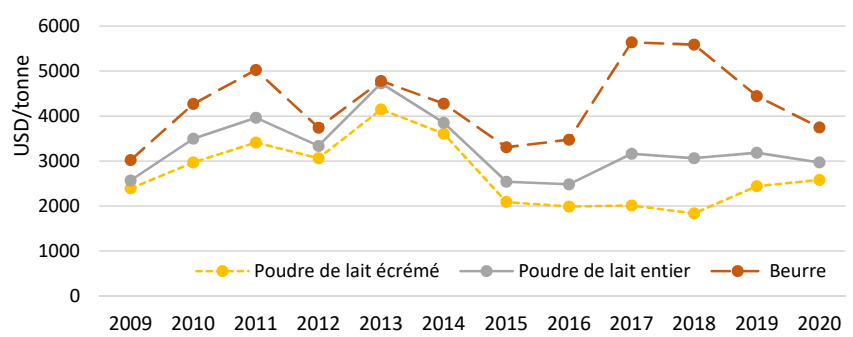

Figure 1 : Prix internationaux de la poudre de lait et du beurre (FAO, 2020b) /// International prices of milk powder and butter (FAO, 2020b) 


\section{Démarche méthodologique}

En nous basant sur Gouin et Kroll (2018) nous avons opté pour une méthode consistant à présenter un historique des différentes mesures et mécanismes de régulation laitière à travers une revue de la littérature. Les politiques laitières algériennes sont analysées sous un angle chronologique en séparant pour chaque période les politiques d'appui à la production locale et les politiques commerciales.

Il s'agit d'abord de comprendre comment la politique commerciale permet au pays de gérer les déséquilibres de marché par des subventions aux importations, et comment ces politiques commerciales aboutissent à un partage de la plus-value des produits entre Etat, industriels, producteurs et consommateurs. Dans le cas de l'Algérie, en effet, les politiques commerciales laitières ont été très fortement marquées par le souci d'approvisionner les consommateurs en produits de base bon marché, quitte à en répercuter le prix sur le budget de l'Etat. Il s'agit ensuite de comprendre en quoi les politiques d'appui à la production s'appuient sur des mécanismes de subvention et d'aide à l'investissement dans la production et la collecte ainsi que sur des mesures de prix bonifiés.

Au fil du texte, nous présentons les institutions en charge de gérer les modalités d'intervention publique dans la gestion du marché, et leurs transformations historiques. Après cet historique, nous évaluons la cohérence de ces interventions, ou les contradictions qu'elles soulèvent, et les impacts qu'elles ont eus sur le profil actuel du secteur laitier algérien. Enfin, nous discutons des moyens de lever ces contradictions.

\section{POLITIQUES DE REGULATION DU SECTEUR LAITIER DEPUIS 1962}

\section{Création de l'Onalait au lendemain de l'Indépendance}

Dès l'Indépendance de l'Algérie en 1962, l'Etat a adopté une série de mesures d'intervention dans l'ensemble des secteurs agricoles afin d'assurer la couverture des besoins alimentaires de la population puisqu'elle n'a hérité d'aucune installation performante sur le plan technique et économique, sauf pour de petites structures et des fromageries mises en place par le secteur privé et dont la propriété est liée à des groupes français d'exportation. Ces laiteries privées au nombre de six étaient des centres de conditionnement du lait pasteurisé importé par les firmes françaises Gopexalait et Pyrénées Lait. Pour le lait, l'Etat crée en 1969 l'Office national du lait (Onalait) chargé de mettre en place la politique publique de régulation du secteur. Il s'agit pour cet office d'encourager la structuration et l'encadrement de la production bovine laitière dans les exploitations agricoles. Il s'agit aussi d'encourager l'intégration industrielle de la production laitière locale afin d'assurer une intensification du système de production capable de se substituer progressivement à la poudre de lait importée. Toutefois, l'accroissement de la demande en produits laitiers et la hausse des prix du pétrole ont entrainé un délaissement progressif de la production locale en faveur de l'importation de la poudre facile à reconstituer dans les installations industrielles publiques. Ainsi, entre 1970 et 1980, la collecte de lait par l'Onalait passe de 41 à 43 millions de litres, ce qui représente une progression insignifiante. Dans le même temps, l'Office cesse toutes les importations de lait en vrac, qui étaient de l'ordre de 15 millions de litres en 1970, et les remplace par des importations de poudre de lait et de matière grasse de lait anhydre qui s'envolent pour atteindre 378 millions de litres en 1980 (Kherzat, 2007). Ainsi, l'objectif global de sécurité alimentaire se fonde dans les années qui suivent l'Indépendance sur la priorité donnée à l'importation continue de poudre de lait.

En 1983, l'Onalait est restructuré en trois offices régionaux qui gèrent au total 17 unités de transformation publiques dépendantes. L'activité essentielle de ces unités reste la transformation de la poudre importée pour la production de produits de grande consommation financée par la rente pétrolière. De manière délibérée, les offices régionaux se désengagent de la production laitière locale, avec l'objectif affiché de mettre à disposition des consommateurs des produits « stratégiques » à bas prix (Bencharif, 2001 ; Giplait, 2010). Le secteur laitier algérien se construit alors sur une situation paradoxale où les prix à la consommation deviennent inférieurs aux prix à la production (Kherzat, 2007).

Pour appuyer le secteur, le gouvernement met en place divers autres offices destinés à garantir l'accès des exploitations aux intrants. Là encore, ces offices vont promouvoir les importations. C'est le cas par exemple de l'Office national d'approvisionnement et de services agricoles (Onapsa), destiné à faciliter l'importation de produits vétérinaires et de matériel génétique, et de l'Office national d'aliment du bétail (ONAB) qui va contribuer à renforcer l'importation de matières premières alimentaires (Bedrani et al., 1997).

Cette stratégie de sécurité alimentaire basée sur les prix administrés et sur les subventions aux importations est maintenue malgré plusieurs crises, notamment le contre-choc pétrolier de 1986, associant pénuries et hausses des prix sur le marché mondial et suggérant déjà les limites de cette stratégie de recours aux importations. Au cours de ces années, la facture d'importation de poudre de lait représente une charge financière croissante pour l'Etat. Sa valeur cumulée entre 1982 et 1992 atteint $15 \%$ du volume de la dette extérieure en 1992 (Amellal, 1995).

\section{Programmes de relance de la production laitière (1995-2009)}

Devant les difficultés rencontrées pour promouvoir la production laitière locale, le ministère en charge de l'Agriculture conduit dès 1992 une série d'études et de réformes dans le secteur laitier qui aboutissent à plusieurs programmes de relance de la production laitière. Lancé en 1995, le Programme de réhabilitation de la production laitière est la première grande action politique dans ce domaine (Cherfaoui et al., 2004). Il vise le développement de la production nationale de lait cru et l'augmentation du taux d'intégration de la production nationale dans l'industrie laitière. Il se base sur deux outils principaux qui resteront au cœur des politiques laitières algériennes jusqu'à nos jours : a) des subventions aux investissements qui concernent les fermes, les laiteries, les matériels de collecte et la capacité d'insémination artificielle ; et b) des bonifications du prix du lait aux éleveurs et aux collecteurs, qui protègent les revenus des acteurs de la filière tout en permettant le maintien des bas prix aux consommateurs. Le gouvernement attribue ainsi des primes de 4 dinars algériens (DA ; 1 DA $\approx 0,0086$ US\$) par litre aux éleveurs, et de $2 \mathrm{DA} / \mathrm{L}$ aux collecteurs. Ces subventions et bonifications sont attribuées via des fonds spéciaux (tableau I).

Pour appuyer la mise en œuvre de ce programme, le Conseil interprofessionnel du lait (CIL) et l'Office national interprofessionnel du lait (ONIL) ont été créés en 1997. Le CIL fédère l'ensemble des opérateurs de la filière sans pour autant permettre une meilleure représentation des producteurs dans la gouvernance de la filière, alors que l'ONIL est chargé d'organiser les marchés agricoles du lait et produits laitiers, et de mettre en œuvre les politiques d'incitation au développement de la production laitière en concertation avec le CIL. L'ONIL s'imposera petit à petit jusqu'à jouer pleinement son rôle à partir de 2007. Il contrôle désormais la distribution des quotas de la poudre de lait importée et des primes dites d'intégration du lait local dans la transformation industrielle (tableau I) (Makhlouf et Montaigne, 2016).

Du côté des industries de transformation, le programme de réhabilitation de la production laitière conduit à la fusion des 14 (puis 15) unités 
Tableau I : Répartition de l'octroi de prime selon le programme de soutien agricole (Algérie) /// Distribution of an incentive payment according to the agricultural support program (Algeria)

\begin{tabular}{|c|c|c|c|c|}
\hline \multirow[t]{2}{*}{ Année } & \multirow[t]{2}{*}{ Programme de soutien agricole } & \multicolumn{3}{|c|}{ Prime (DA/L) } \\
\hline & & Prod. & Coll. & Tran. \\
\hline 1995 & $\begin{array}{l}\text { Programme de réhabilitation } \\
\text { de la production du lait cru par } \\
\text { le biais du Fonds national de } \\
\text { développement agricole (FNDA) }\end{array}$ & 4 & 2 & 0 \\
\hline 2000 & $\begin{array}{l}\text { Programme national de } \\
\text { développement agricole (PNDA) } \\
\text { (création du Fonds national de } \\
\text { régulation et développement } \\
\text { agricole [FNRDA] devenu FNRPA) }\end{array}$ & 7 & 4 & \\
\hline 2009 & Renouveau agricole et rural (RAR) & 12 & 5 & 2 à $4^{*}$ \\
\hline $\begin{array}{l}\text { Depuis } \\
2015\end{array}$ & $\begin{array}{l}\text { (rentorcement du FNRPA par le } \\
\text { Fonds national de développement } \\
\text { de l'investissement agricole } \\
\text { [FNDIA]) }\end{array}$ & 14 & & 2 à 6 * \\
\hline
\end{tabular}

DA : dinars algériens ; Prod. : production ; Coll. : collecte ; Tran. : transformation. * Selon le taux d'intégration adopté par la laiterie //I DA: Algerian dinars; Prod.: production; Coll.: collection; Tran.: transformation. ${ }^{*}$ Depending on the integration rate adopted by the dairy

de transformation gérées par les anciens offices régionaux pour créer le Groupe industriel des productions laitières (Giplait). Cependant, entre 1996 et 2001, la part de la collecte de lait cru dans l'approvisionnement de cette société d'Etat chute fortement de 140 à 90 millions de litres par an (Kherzat, 2007). Ces unités ont échappé à la privatisation amorcée dans le secteur public dès les années $2000 \mathrm{vu}$ leur caractère stratégique. Depuis, elles continuent d'exercer un rôle important notamment dans la transformation et la distribution du lait reconstitué.

Pendant cette période, toutefois, les politiques commerciales restent les mêmes et le secteur industriel continue à s'appuyer sur l'importation de poudre à prix subventionné. Entre 1992 et 2010, les quantités importées enregistrent une augmentation moyenne de 2,4\% par an en volume et de 5,4\% par an en valeur. Sous l'effet de la croissance démographique (+29,8\% cumulés sur la période) et de la hausse des cours sur le marché mondial, la facture d'importation double entre 1992 et 2008 (Mamine, 2014).

\section{Politique du Renouveau agricole et rural (depuis 2009)}

En 2009, en réaction à la crise financière et économique et aux prémices du Printemps arabe, le ministère de l'Agriculture et du Développement rural (MADR) lance la politique du Renouveau agricole et rural (RAR) pour renforcer le développement des filières stratégiques dont la filière lait local. De nouveaux programmes de subvention à la filière sont alors mis en place (figure 2) avec la poursuite des subventions à l'investissement, une augmentation des primes à la production et à la collecte, et la création de primes aux transformateurs pour l'intégration industrielle du lait local (tableau I).

\section{Subventions et primes aux éleveurs}

Les subventions aux investissements concernent l'équipement de l'exploitation, la modernisation des étables, l'achat de matériel de traite et de réfrigération, et l'achat de génisses. S'ajoutent ensuite les subventions à l'achat des facteurs de production que sont a) l'aliment du bétail, issu principalement de matière première importée, b) les fourrages, avec une aide aux semences et aux moyens de conservation, et c) l'insémination artificielle. Les producteurs reçoivent également une prime de l'ONIL qui constitue un complément de revenu, mais uniquement s'ils vendent leur lait aux industriels en respectant

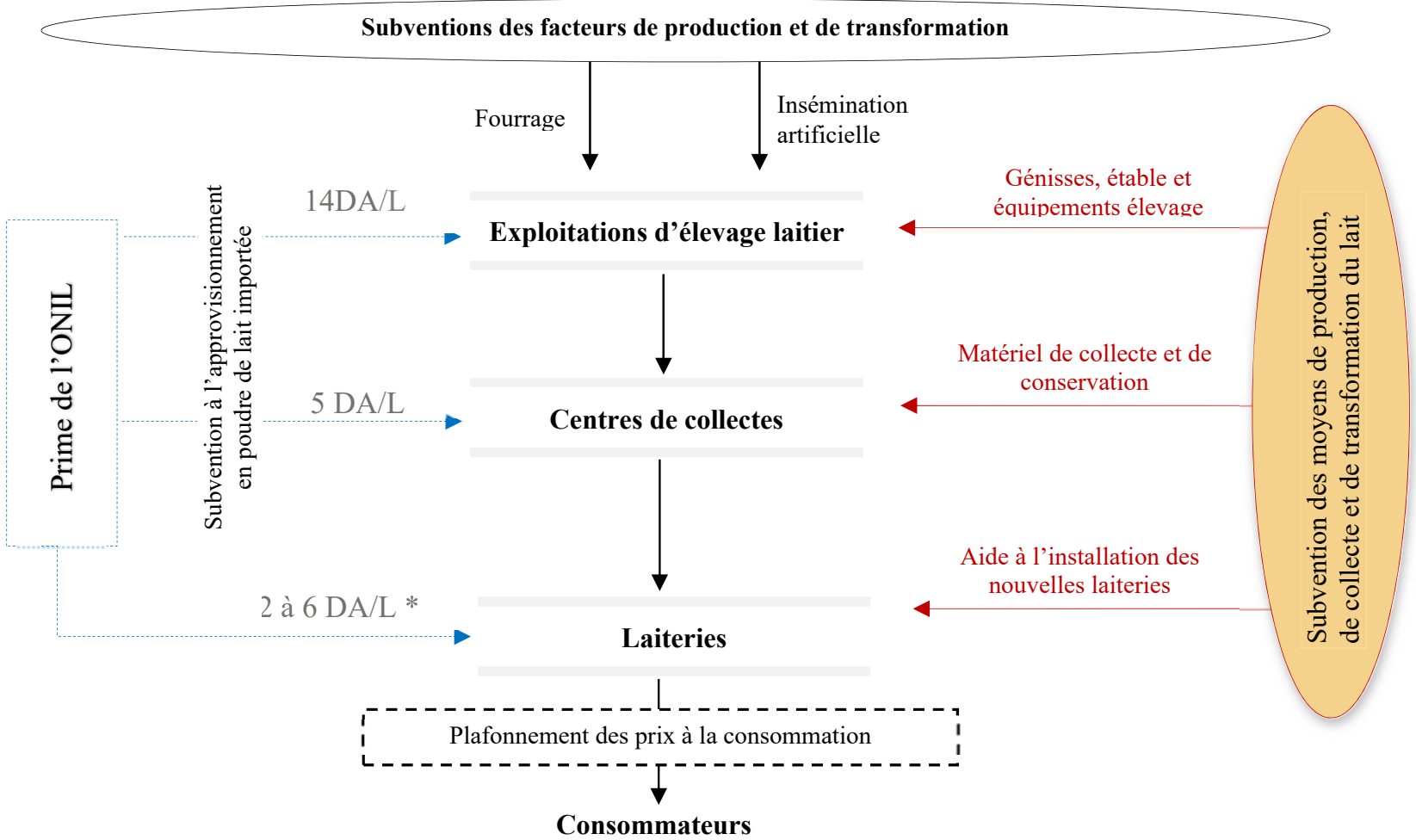

Figure 2 : Interventions publiques dans la filière lait en Algérie.* Prime de 6 DA/L si la laiterie ne fonctionne qu'avec le lait local /// Public interventions in the milk sector in Algeria. ${ }^{*} 6 \mathrm{DA} / \mathrm{L}$ incentive payment if the dairy operates only with local milk 
les règles sanitaires. Ces bonifications s'élèvent à 12 DA/L en 2012, et sont abondées d'une surprime de $2 \mathrm{DA} / \mathrm{L}$ à partir de fin 2015 pour les élevages répondant au suivi sanitaire (agrément sanitaire contre la tuberculose et la brucellose). La revalorisation de ces primes a pour objectif de réduire l'écart de prix avec la poudre de lait fortement subventionnée (tableau I).

Ces aides sont cependant accordées conditionnellement à une taille de l'exploitation : un effectif minimal de 12 vaches laitières (VL) et une surface de six hectares de terre en propriété. Cette règle d'accès à la subvention a été allégée en 2009 (6 VL et 5 ha en propriété ou en location). Selon les chiffres du ministère de l'Agriculture en 2015, seuls 34300 sur les 300000 éleveurs bénéficiaient de primes à la production, ce qui montre l'exclusion de la majorité des petits éleveurs qui ne peuvent remplir les conditions d'octroi des subventions publiques. Dans le cadre du RAR, l'Etat met aussi en place un mécanisme de prix minimum garanti pour soutenir la production de lait local. Ce prix minimum « à la sortie de la ferme » est passé de 30 DA/L en 2012 à 36 DA/L en 2016.

\section{Subventions et primes aux collecteurs}

Le collecteur, maillon important qui s'occupe de la collecte du lait auprès des producteurs et qui assure son transfert aux transformateurs, reçoit deux types d'aides : a) une subvention à l'équipement, achat de camions ou de pick-up équipés d'une citerne isotherme, et b) une prime incitative, proportionnelle à la quantité collectée $(5 \mathrm{DA} / \mathrm{L}$ depuis 2012). Ces aides permettent le développement d'une activité importante de ramassage quotidien du lait auprès de nombreuses petites exploitations isolées. Ces collecteurs ou ramasseurs agréés par l'ONIL, au nombre de 1600 en 2017, sont majoritairement indépendants ou rattachés aux centres de collecte ou, rarement, à des laiteries.

Les prix à la sortie d'exploitation sont en principe fixes puisque définis par l'ONIL mais en raison de l'incapacité des éleveurs à assurer les indicateurs de qualité exigés par les industriels, ils sont souvent négociés à la baisse par l'intermédiaire des collecteurs. Pour valoriser le lait non conforme rejeté par les industriels, les collecteurs alimentent souvent les circuits informels non agréés à travers la vente directe auprès des consommateurs et la vente auprès des transformateurs artisanaux.

\section{Subventions aux industriels}

Pour encourager l'intégration du lait local dans les circuits de collecte industriels, les laiteries peuvent recevoir une prime dite d'intégration du lait local qui est de 2 à 6 DA/L (tableau I). Cette intégration vise à encourager l'élaboration de produits transformés à base de lait local, comme les fromages et les yaourts, qui sont alors commercialisés à des prix libres. Le lait local se vend de 50 à $70 \mathrm{DA} / \mathrm{L}$ dans les grands centres urbains. Cependant, dans la pratique, seules 27 des 135 laiteries conventionnées avec l'ONIL ont choisi de s'approvisionner exclusivement en lait local.

\section{Maintien des politiques de sécurité alimentaire en faveur des consommateurs}

Subventions aux importations et prix administrés à la consommation

Afin de garantir l'accès des consommateurs à des produits laitiers de base et parallèlement aux programmes d'appui à la production, l'Etat algérien poursuit ses politiques de soutien à la consommation. Il renouvelle ses programmes de subventions aux importations et de prix administrés à la consommation. A partir de 2007, c'est l'ONIL qui est chargé de l'approvisionnement des laiteries en poudre de lait importée destinée à la reconstitution en lait standardisé. L'Office cède la poudre de lait écrémé importée à 159000 DA/tonne, quel que soit son prix d'achat sur le marché mondial, et l'Etat prend en charge la différence entre ce prix de vente et le prix d'achat réel. A ce prix, la poudre de lait nécessaire à la production d'un litre de lait reconstitué revient à environ 15,9 DA/L. Par ailleurs, le prix à la consommation du lait pasteurisé en sachet (LPS) est à $25 \mathrm{DA} / \mathrm{L}$. Les 120 laiteries conventionnées avec l'ONIL (15 unités publiques et 105 privées) s'engagent donc à vendre le lait reconstitué à un prix plafonné à 23,5/L DA à la sortie de l'usine et à le distribuer selon une quantité moyenne journalière correspondant aux quantités affectées par l'Office. Entre 2007 et 2010, l'Etat a injecté plus de 32,2 milliards de DA pour couvrir la différence entre le prix de la poudre de lait achetée sur le marché mondial et le prix de revente sur le marché national.

\section{Mise en place d'un stock stratégique de poudre de lait}

Pour atténuer l'impact de la forte volatilité du prix des produits alimentaires, le gouvernement algérien a toutefois mis en place un outil de régulation des marchés appelé Système de régulation des produits agricoles de large consommation (Syrpalac). Le seul apport tangible de ce mécanisme de régulation réside dans le renforcement de la capacité de stockage de poudre importée pour couvrir jusqu'à six mois de consommation. Cependant, cette mesure de court terme ne peut permettre de faire face à l'évolution de la demande de lait. Un renforcement des capacités d'offre sur le marché local passe nécessairement par le développement d'une filière de lait local.

En 2014, la consommation totale est estimée à environ six milliards de litres EL, dont trois milliards proviennent de l'importation, pour une valeur de 1,4 milliard US\$. Sur une production locale totale de 3,4 milliards litres par an (environ $56 \%$ des besoins nationaux), seulement 0,9 sont collectés pour la transformation en industrie, le reste étant commercialisé par d'autres circuits ou autoconsommé. De ce fait, le lait local n'assure qu'environ $25 \%$ des besoins de l'industrie laitière dont la capacité totale s'élève à environ 3,4 milliards de litres EL (Hirondel, 2014). En 2018, la valeur des importations est restée la même et s'élevait à 1,4 milliard US\$ (DPE, 2020).

\section{- IMPACT DE CES PROGRAMMES}

\section{SUR LES ACTEURS DU SECTEUR}

\section{Caractéristiques et performances des exploitations}

La modernisation technique de l'élevage bovin est la priorité de la politique de développement du lait local. Cependant, la gestion administrée des grandes fermes, mises en place dans les années 1970 par le régime socialiste, a résulté en des choix techniques inefficaces. L'échec de l'installation des grandes fermes dans des zones de forte concurrence sur les ressources foncières et hydriques constitue un exemple parmi d'autres des obstacles rencontrés par cette gestion administrée de la production (Benfrid et al., 1993). L'abandon de cette politique administrée et le choix de la privatisation des exploitations agricoles ont permis la création d'un grand nombre d'exploitations laitières privées après le démembrement des anciens domaines d'Etat (MADR, 2003). Toutefois, plus de 55,3\% des exploitations ont moins de cinq hectares et de ce fait ne sont pas éligibles aux subventions publiques. Une grande partie de ces petites exploitations ont ainsi conservé leur mode de fonctionnement traditionnel avec des circuits informels de vente et hors des circuits industriels subventionnés.

La production laitière bovine représente environ $73 \%$ de la production laitière nationale à côté des productions laitières caprines, ovines et camelines (communiqué de presse du ministère de l'Agriculture et du Développement rural). Cependant, le lait de vache représente la quasi-totalité du lait commercialisé car le lait produit par les autres espèces est destiné soit à l'allaitement des nouveau-nés soit à l'autoconsommation.

Depuis l'Indépendance, la production laitière bovine affiche un taux de croissance annuel moyen de $5,4 \%$ du fait d'une augmentation du cheptel de 2,7\% par an et d'une amélioration du rendement moyen 
par vache de 2,7 \% par an. Pour améliorer la production laitière locale, l'Etat a misé sur l'importation de génisses pleines à haut potentiel génétique. Toutefois, le rendement moyen annuel s'élève seulement à $2100 \mathrm{~L} /$ vache (6300 L/vache en France ; CNIEL, 2008), toutes races comprises (Giner, 2010). Ce rendement relativement limité s'explique par : a) la diversité des systèmes d'élevage, avec une surreprésentation des systèmes extensifs avec un faible cheptel (le système intensif peine à se développer en raison d'une disponibilité limitée des ressources foncières et en eau) ; b) la lenteur de la modernisation de l'élevage laitier par insuffisance d'investissements techniques et humains ; c) la préférence des éleveurs pour la valorisation de la production laitière par l'allaitement des veaux (nous estimons la valeur de la rente bouchère d'un litre d'EL à environ 151 DA contre 36 DA le prix moyen payé sur le marché) ; d) le manque d'incitations à la production et la faiblesse du prix minimum garanti, couvrant à peine la hausse des coûts de production; et e) des ressources naturelles limitées du fait d'un climat semi-aride à aride sur plus de $90 \%$ de la surface du pays, ce qui est un handicap majeur pour la production fourragère qui nécessite des ressources hydriques importantes.

\section{Progrès génétiques insuffisants du cheptel laitier}

Les pouvoirs publics ont surtout misé sur l'importation du matériel génétique vivant (génisses pleines à haut potentiel génétique de races Holstein, Montbéliarde, Fleckvieh, Brune des Alpes...), favorisée par un droit de douane faible (5\%). Entre 2011 et 2012, 54800 génisses ont été importées (Soukehal, 2013). L'effectif moyen importé est variable selon la disponibilité de la manne pétrolière et depuis 2013 le nombre de génisses importées a été revu à la baisse. Leur importation reste insuffisante par rapport à la demande, malgré une politique continue d'importation à partir du milieu des années 1960 menée par le Centre national d'insémination artificielle et d'amélioration génétique (CNIAAG), puis par des opérateurs privés depuis le début des années 2000 (CN AnGR, 2003). En outre, la pertinence de cette stratégie peut être questionnée du fait des performances zootechniques relativement limitées de ces races importées (Madani et Far, 2002 ; Madani et Mouffok, 2008)

Plusieurs facteurs ont contribué à la faible efficacité de ces politiques d'importation de génisses : a) l'absence d'un schéma de sélection et d'un appareil effectif de gestion de l'amélioration et de la sélection génétique ; b) la réticence à l'adoption de l'insémination artificielle dans l'élevage laitier malgré les subventions accordées par l'Etat dans ce domaine (75\% du coût total) et l'assistance technique assurée par le CNIAAG ; c) le manque d'adaptation à leur nouvel environnement d'un grand nombre d'animaux importés conduisant à leur réforme et abattage précoce afin de tirer profit de leur valeur bouchère jugée plus rémunératrice. Dans certaines régions du pays, le taux de réforme des vaches laitières peut atteindre plus de $43 \%$ durant les trois premières années de l'élevage (Benallou et al., 2011).

\section{Production fourragère insuffisante}

Les exploitations d'élevage laitier n'arrivant pas à produire leurs propres intrants sont en effet presque entièrement dépendantes de l'importation de la matière première (maïs, tourteau de soja) utilisée dans la fabrication d'aliments composés. L'affectation par les éleveurs d'une part réduite de la sole de l'exploitation à la production fourragère (concurrence avec les autres cultures, particulièrement les céréales) et les difficultés de l'intensifier en raison d'un régime pluviométrique aléatoire expliquent la faible autonomie alimentaire des exploitations d'élevage bovin laitier (Nedjraoui, 2001).

Pour pallier ce déficit, l'Etat s'est engagé dans le développement de cultures fourragères afin d'assurer l'intensification de l'élevage laitier. Il s'agit de cultures de céréales (orge, avoine, triticale) majoritairement en zones pluviales, de mélange de céréales avec des pois ou vesces, alors que le maïs, le sorgho et la luzerne dominent en irrigué en saison estivale avec des besoins hydriques très élevés. Dans ce domaine, l'Etat a alloué 98,75 millions de DA entre 2000 et 2010. Depuis, la production fourragère a sextuplé (MADR, 2012). D'après les données statistiques plus récentes du ministère de l'Agriculture (MADR, 2017), la suppression de cette subvention (figure 3) ne semble pas freiner le développement de ces cultures (49 millions de quintaux en 2016 contre 4 millions de quintaux à l'aube des années 2000). Toutefois et en raison de leur caractère pluvial dominant, leur rendement reste limité (en moyenne 2,46 t/ha) par rapport au fourrage naturel issu des prairies naturelles ou des jachères fauchées (en moyenne 3,1 t/ha) (MADR, 2017).

\section{Profil des acteurs de l'aval de la filière}

L'augmentation du taux d'intégration industrielle du lait local, défini comme le ratio entre la quantité de lait local transformé et la quantité totale de lait transformé, représentait théoriquement pour l'Etat un objectif important qui devait se traduire par le développement de l'élevage laitier local. L'Etat a donc accordé au début des années 1970 un intérêt particulier à la transformation industrielle du lait local et à ses circuits de distribution dans les grandes agglomérations urbaines.

\section{Collecte industrielle insuffisante}

Le taux de collecte industrielle du lait local s'est rapidement effondré dans les années 1970 lorsque se sont développées de grandes usines de transformation de la poudre de lait importée (figure 4). Cette situation d'une filière de lait local marginalisée sur le plan industriel s'est maintenue jusqu'à la moitié des années 1990. La difficulté d'accès à la transformation industrielle du fait de la concurrence de la poudre de lait a eu pour conséquence l'émergence de circuits informels sous la forme de vente directe de lait ou de produits laitiers transformés artisanalement.

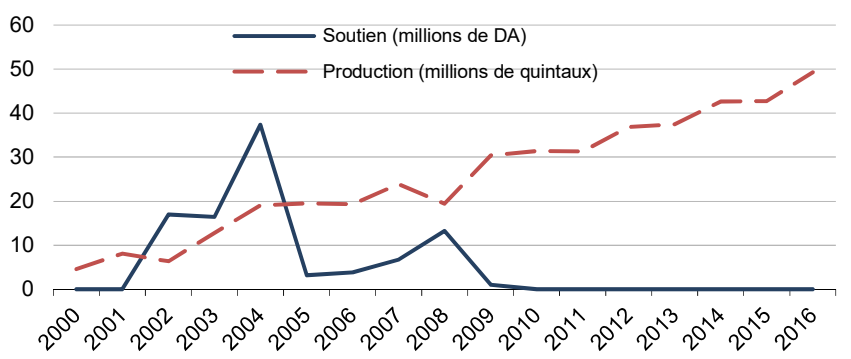

Figure 3 : Soutien de l'Etat algérien accordé au développement du fourrage artificiel; DA : dinars algériens /// Algerian State support for the development of artificial fodder; DA: Algerian dinars

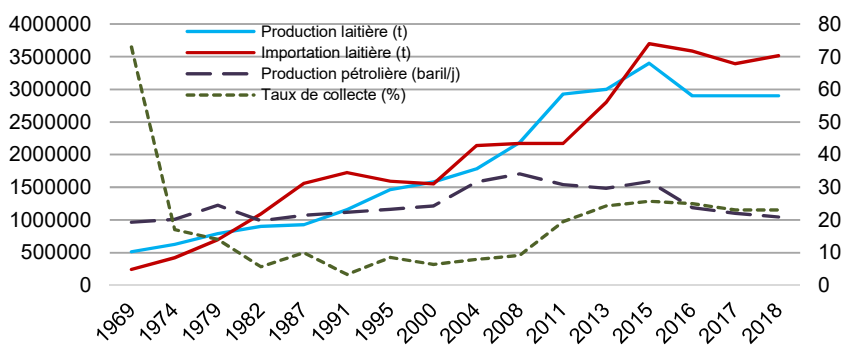

Figure 4 : Evolution de la production, du taux de collecte et de l'importation de lait par rapport à la production de pétrole. Synthèse des auteurs d'après les données de Cherfaoui, 2003 ; MADR, 2012 ; FAO, 2020 ; www.eia.gov //I Evolution of milk production, collection rate and import in relation to oil production. Authors' summary based on data from Cherfaoui, 2003; MADR, 2012; FAO, 2020; www.eia.gov 


\section{Limites d'une transformation industrielle basée sur la poudre importée}

La capacité de transformation a certes connu une augmentation spectaculaire à la suite de la restructuration du secteur public au cours des années 1980, permettant l'apparition de nouveaux acteurs privés. En 2012, la filière englobe 15 entreprises du groupe public Giplait et plus de 162 entreprises privées dont la capacité de transformation est très variable (Makhlouf et Montaigne, 2016). Du fait de la subvention à l'importation de la poudre de lait, les industriels n'avaient aucun intérêt à développer la collecte du lait local (figure 5). Cependant la hausse des prix sur le marché mondial en 2008 a entraîné une augmentation importante du prix de la poudre de lait, rendant concurrentielle la production locale de lait. Cette crise a mis en lumière les limites d'un modèle laitier en grande partie fondé sur des approvisionnements externes, et une absence criante de résilience face aux transformations profondes de l'environnement externe et du marché mondial.

Par le biais du programme RAR, l'Etat a injecté des sommes importantes pour favoriser la collecte et la transformation industrielle du lait local (plus de 3,2 milliards de DA en 2009 et plus de 4,3 milliards en 2011) (ONIL, 2012). Si le taux de collecte a doublé entre 2008 et 2011 (figure 4) sous l'impulsion des diverses primes à la production et à la collecte (tableau I), il n'en demeure pas moins que le taux d'intégration ne dépasse pas actuellement $20 \%$ de la production alors qu'il était estimé à plus de $70 \%$ au début des années 1960. Si cet ensemble de mesures tend à orienter le fonctionnement du secteur laitier vers un modèle industriel, il ne tient pas compte de la structure de l'amont de la filière qui se caractérise par une offre atomisée et saisonnière, un lait de qualité médiocre, ce qui la rend peu compatible avec les stratégies des laiteries.

\section{Gestion de la qualité encore peu compatible avec le modèle industriel}

En amont de la filière, les exploitations d'élevage laitier disposent de peu de moyens techniques pour une alimentation suffisante et équilibrée de leur cheptel pour une traite mécanisée et une réfrigération rapide du produit. Les compétences nécessaires à la maîtrise du rendement par vache et la qualité du lait font défaut. Par ailleurs, l'attribution des primes à la production n'est plus conditionnée au respect du contrôle sanitaire depuis 2009, ce qui a entrainé la baisse du nombre d'élevages soumis à l'agrément sanitaire, et ce, malgré la revalorisation de ladite prime qui est passée de 12 à $14 \mathrm{DA} / \mathrm{L}$ en 2015. La Chambre nationale d'Agriculture estime que plus de $80 \%$ des exploitations d'élevage laitier ne sont soumises à aucun contrôle sanitaire. De plus, le manque de traçabilité dans l'opération de collecte (mélange du lait issu des exploitations contrôlées avec celui de celles non contrôlées) s'ajoute à la mauvaise gestion de la qualité en amont de la filière.

En aval de la filière, la fonction officielle des centres de collecte est de livrer aux industriels le lait cru produit par les éleveurs avec lesquels ils ont des contrats spécifiant les attributs de qualité physicochimique qui leur est exigée (densité, acidité, taux de matière grasse). En effet, tout lait non conforme à ces exigences qualitatives, surtout le lait caillé, est systématiquement rejeté par les industriels ce qui contribue au faible taux d'intégration industrielle du lait local. Cela explique le maintien des circuits informels du lait et de sa transformation artisanale, pour lesquels les niveaux exigés de qualité physicochimique sont moins contraignants qu'en transformation industrielle, notamment les petites crèmeries aux moyens techniques limités (caillage, barattage, fermentation) sans pasteurisation. Elles produisent principalement du Raëb (lait caillé), du Lben (petit lait) et du beurre de ferme, produits qui demeurent cependant très prisés par la population locale (Mamine et al., 2016).

\section{CONCLUSION}

Dans le secteur laitier en Algérie, l'Etat intervient par des mécanismes de régulation dans l'objectif d'assurer l'équilibre du marché. Ces mécanismes encadrant la filière lait soutiennent l'ensemble des opérateurs à différents niveaux mais avec des résultats controversés. Sur le plan de l'élevage bovin, les efforts fournis dans la promotion de l'investissement technique ne permettent pas une structuration efficace des systèmes de production pour plusieurs raisons : a) l'insuffisance des moyens financiers mobilisés dans la modernisation technique ; b) la mauvaise répartition du soutien public dans la filière lait car seuls $11,4 \%$ des éleveurs sont éligibles à l'aide de l'Etat ; c) la faible intégration et valorisation de la production locale dans la transformation laitière ( $20 \%$ ) ; et d) le différentiel de prix entre prix du lait local et prix de la poudre de lait, principalement dû à la subvention de la poudre de lait importée.

Le recours à l'importation est vu comme nécessaire pour sécuriser l'approvisionnement du marché national des produits laitiers. La filière laitière locale a de ce fait du mal à décoller, ce qui se traduit par sa faible intégration dans la transformation laitière. En fait, l'amélioration de la collecte et de l'intégration industrielle n'est perceptible que depuis les années 2011 et 2012. L'ajustement du marché par les politiques de prix minimum garanti et de primes n'a pas permis de gommer le différentiel entre prix du marché local et prix du marché international. L'encadrement de l'élevage laitier a permis cependant certaines améliorations des performances de la filière lait avec la mise en œuvre des différents programmes du développement agricole. L'intervention de l'Etat a contribué à l'extension du marché de consommation (plafonnement des prix), mais sans un accroissement suffisant de la production laitière locale qui repose principalement sur des exploitations d'élevage de taille réduite. Le développement récent des mégafermes dans la zone steppique peut contrebalancer cette configuration s'il est associé à une gestion durable des ressources hydriques puisées dans les réserves souterraines (objectifs spécifiques définis par l'Unesco pour développer une gestion durable des eaux souterraines ${ }^{1}$ ). Pour réussir ce modèle industriel, les pouvoirs publics peuvent également tirer des leçons managériales de l'échec des grandes fermes du domaine public de l'époque socialiste.

1. https://fr.unesco.org/themes/securite-approvisionnement-eau/hydrologie/eauxsouterraines/gestion-durable)

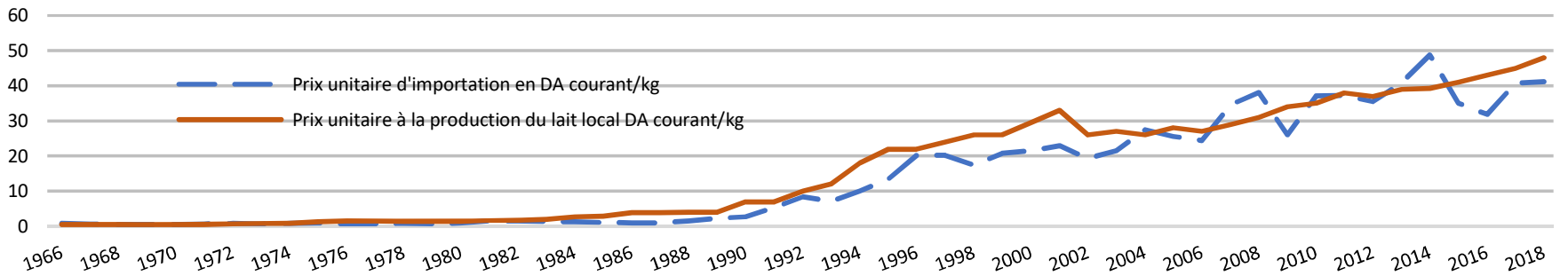

Figure 5 : Evolution des prix du lait à la production et à l'importation (équivalents lait) en Algérie /// Evolution of milk prices at production and import (milk equivalents) in Algeria 
La poudre de lait pèse de plus en plus sur la facture extérieure dans un contexte mondial caractérisé par une crise alimentaire et l'instabilité des prix des hydrocarbures, principale source de devises pour garantir la sécurité alimentaire en Algérie.

L'objectif des politiques publiques de la filière lait est en priorité la sécurisation de l'approvisionnement et la satisfaction de la demande des consommateurs. L'importation de la poudre de lait et le développement de la production laitière locale constituent des alternatives stratégiques vis-à-vis desquelles l'Etat doit constamment effectuer des arbitrages. Les résultats escomptés, à savoir l'augmentation du taux de couverture de la consommation intérieure par la production et le développement harmonieux de bassins laitiers dans les territoires ruraux du pays, sont encore loin d'être atteints. La récurrence des crises d'approvisionnement nationales en lait remet en question l'efficacité du système de régulation de cette filière mise en place par les pouvoirs publics et la fiabilité des données sur lesquelles elle s'appuie pour assurer la couverture des besoins du marché national en produits laitiers. Le besoin de développer la production locale en facilitant davantage l'accès des petits producteurs au marché et aux différentes formes de soutien public de la filière répond non seulement à l'objectif de réduire la dépendance du pays aux importations, mais aussi de donner les moyens au secteur agricole de contribuer à la diversification et à la dynamisation de l'économie nationale, très dépendante des exportations des hydrocarbures.

\section{Remerciements}

Les auteurs remercient les agents de l'INRAA d'Alger pour leur collaboration dans la phase de collecte des données.

\section{Conflits d'intérêts}

Létude a été réalisée sans conflit d'intérêts.

\section{Déclaration des contributions des auteurs}

FM et MF ont conçu et planifié l'étude ; FM a assuré notamment la collecte, l'analyse et l'interprétation des données puis avec l'aide de $\mathrm{MF}$ a rédigé la première version du manuscrit. GD et TM ont révisé et complété la rédaction du manuscrit.

\section{REFERENCES}

Amellal R., 1995. La filière lait en Algérie: entre l'objectif de la sécurité. In: Allaya M. (ed.). Les agricultures maghrébines à l'aube de I'an 2000. Montpellier (France): CIHEAM Options Méditerranéennes: Série B, Etudes et recherches 14: 229-238

Baritaux V., Chatellier V., Trouvé A., 2018. La libéralisation des marchés laitiers. Quelles réponses des acteurs économiques et des politiques? Econ. Rurale, 364: 7-12, doi: 10.4000/economierurale.5484

Bedrani S., Boukhari N., Djennane A., 1997. Eléments d'analyse des politiques de prix, de subvention et de fiscalité sur l'agriculture en Algérie. In: Akesb N., Maraveyas N., eds, Prix et subventions : effets sur les agricultures familiales méditerranéennes. CIHEAM Options Méditer. Sér. B, 11: 121-149

Benallou B., Kouidri M., Ghazi K., 2011. Evaluation des performances de reproduction de la vache laitière dans la région de Tiaret. Rev. Ecol. Environ., 7: 27-35

Bencharif A., 2001. Stratégies des acteurs de la filière lait en Algérie: état des lieux et problématiques. In : Padilla M., Ben Saïd T., Hassainya J., Le Grusse P. (eds). Les filières et marchés du lait et dérivés en Méditerranée : état des lieux, problématique et méthodologie pour la recherche. CIHEAM Options Méditer. Sér. B, 32 : 25-45

Benfrid M., Bedrani S., Elloumi M., Zagdouni L., 1993. Schéma et mode de fonctionnement du système de vulgarisation dans les filières avicoles et bovines laitières en Algérie. Montpellier (France). CIHEAM Options Méditer. Sér. B, 2 (1): 123-127

Bouamra-Mechemache Z., Chavas J.P., Cox T., Réquillart V., 2002. EU dairy policy reform and future WTO negotiations: a spatial equilibrium analysis. J. Agric. Econ., 53 (2): 233-257, doi: 10.1111/j.1477-9552.2002.tb00019.x
Chatellier V., 2019. La planète laitière et la place de l'Afrique de l'Ouest dans la consommation, la production et les échanges de produits laitiers. Symp. Lait, Vecteur de Développement, Dakar, Sénégal, Juin 2019

Cherfaoui A., 2003. Essai de diagnostic stratégique d'une entreprise publique en phase de transition : cas de la LFB (Algérie). Thèse Master of Science, CIHEAM-IAMM Montpellier, France, 119 p.

Cherfaoui M.-L., Mekersi S., Amroun M., 2004. Le programme national de réhabilitation de la production laitière : objectifs visés, contenu, dispositif de mise en œuvre et impacts obtenus. Rech. Agron.- INRAA, 15: 84-94

CNIEL, 2008. Les chiffres clés de la filière laitière française en 2008, Paris, France, $16 \mathrm{p}$.

CN AnGR, 2003. Rapport national sur les ressources génétiques animales. Ministère de I'Agriculture et du Développement rural, Algérie / FAO, Italie, $46 \mathrm{p}$.

Corniaux C., Dia D., Diao-Camara A., Duteurtre G., 2011. Les importations laitières : vraie question ou faux problème? Le lait comme révélateur des enjeux du développement agricole au Sénégal, dans un contexte de mondialisation. In: La faim par le marché : aspects sénégalais de la mondialisation (Ed. Pillon P.). L'Harmattan, Paris, France, 219-238

DEP, 2020. Statistiques du commerce extérieur de l'Algérie. Direction des études et de la prospective, Direction générale des douanes, ministère des Finances, $25 \mathrm{p}$.

Diarra A., Benoit-Cattin M., Gérard F., Gabas J.J., Boussard J.M., Duteurtre G., 2013. Echanges internationaux et développement de l'élevage laitier sénégalais. Etude comparative de trois simulations de politique économique. Econ. Rurale, 335: 35-54, doi: 10.4000/economierurale.3936

Duteurtre G., Corniaux C., 2019. Les filières de commercialisation des produits issus de l'élevage des ruminants en Afrique. In Richard D. (Ed.): Dynamique des élevages pastoraux et agro-pastoraux Afrique intertropicale, Quae, Versailles, France, 141-186

Duteurtre G., Pham-Duy K., Cesaro J.-D., 2015. Bassin laitier de Ba Ví : Un territoire d'élevage façonné par les politiques publiques, entre logiques industrielles et soutien à la paysannerie. In: Napoleone M., Corniaux C., Leclerc B. (Eds) : Voies lactées : Dynamique des bassins laitiers entre globalisation et territorialisation. Cardère, Paris, France, 67-87

Duteurtre G., 2008. Panorama des organisations interprofessionnelles dans le monde. In: Duteurtre G., Dieye P.-N. (Eds): Les organisations interprofessionnelles agricoles au Sénégal : de nouveaux outils de régulation des marchés? Isra-éditions, Dakar, Sénégal, 47-52

Duteurtre G., 2009. Lait des pauvres, lait des riches : réflexion sur l'inégalité des règles du commerce international. In: Duteurtre G. Faye B. (Eds), L'élevage, richesse des pauvres : Stratégies d'éleveurs et organisations sociales face aux risques dans les pays du Sud. Quae, Versailles, France, 249-266, doi: 10.3917/quae.duteu.2009.01.0249

FAO, 2020a. Agri-food markets and trade policy in the time of Covid-19. FAO, Rome, Italy

FAO, 2020b. Dairy market Review, FAO, Rome, Italy, 13 p

Giner C., 2010. Nouvelles pistes de création de valeur dans le secteur agroalimentaire. OECD Publishing, Paris, France, 40 p., doi: 10.1787/5kmm$\mathrm{m} x 8 \mathrm{ktfmt}-\mathrm{fr}$

GIPLAIT, 2010. Historique et évolution de l'industrie laitière en Algérie

Gouin D.-M., Kroll J.-C., 2018. La régulation laitière face à la volatilité des marchés-Etats-Unis, Nouvelle-Zélande, Canada, France, Suisse. Econ. Rurale, 364: 13-30, doi: 10.4000/economierurale.5488

Hirondel J.-C., 2014. Le marché de la filière laitière en Algérie 2014. UBIFRANCE, Alger, Algérie, 47 p.

Janin P., De Suremain C.-E., 2005. La question alimentaire en Afrique: risque et politisation. Rev. Tiers-Monde, 4: 727-736, doi: 10.3917/rtm.184.0727

Kherzat B., 2007. Essai d'évaluation de la politique laitière en perspective de l'adhésion de l'Algérie à l'Organisation mondiale du commerce et à la Zone de libre-échange avec I'Union européenne. Mém. magister sciences agronomiques, Institut National Agronomique, El Harrach, Alger, Algérie, $114 \mathrm{p}$

Kroll J.-C., Pouch T., 2012. Régulation versus dérégulation des marchés agricoles: la construction sociale d'un clivage économique. Homme Soc., 1-2 (183-184): 181-206, doi: 10.3917//hs.183.0181

Laroche-Dupraz C.-L., Postolle A., 2010. La souveraineté alimentaire en Afrique est-elle compatible avec les négociations commerciales agricoles à I'OMC? Politique Afr., 3: 107-127, doi: 10.3917/polaf.119.0107

Ledjou R., 2012. The Doha Round and Food Security in the Dairy Sector in Cameroon: A Global Simulation Model (GSIM), Estey Cent. J. Int. Law Trade Policy, 13 (1): 115-130, doi: 10.2139/ssrn.1430821 
Madani T., Far Z., 2002. Performances de races bovines laitières améliorées en région semi-aride algérienne. In: Rencontres Recherches Ruminants, Paris, France, 4-5 déc. 2002

Madani T., Mouffok C., 2008. Milk production and reproductive performance of Montbeliarde cows in a semiarid area of Algeria. Rev. Elev. Med. Vet. Pays Trop., 61 (2): 97-107, doi: 10.19182/remvt.10005

Madelin V., Hébrard L., Scees B., 2007. La flambée des prix des produits laitiers industriels se répercute tardivement sur le prix de lait de vache à la production. Agreste

MADR, 2003. Recensement général de l'agriculture de 2001 : rapport général des résultats définitifs. MADR, Alger, Algérie, $125 \mathrm{p}$.

MADR, 2012. Renouveau agricole et rural en marche : Revue et perspectives. MADR, Alger, Algérie, $58 \mathrm{p}$

MADR, 2017. Statistiques agricoles : surfaces et productions, série B 2016. Doc. travail. Direction des statistiques agricoles et des systèmes d'information. Alger, Algérie, $75 \mathrm{p}$.

Makhlouf M., Montaigne E. 2016. L'impact de la nouvelle politique laitière sur la performance globale de la filière lait en Algérie. In: Coll. SFER Libéralisation des marchés laitiers, Clermont-Ferrand, France, 9-10 Juin, 14 p.

Mamine F., 2014. Rationalité de l'informel : une analyse néo-institutionnelle de la filière lait à Souk Ahras en Algérie. Thèse Doct., Montpellier-SupAgro, Montpellier, France, $423 \mathrm{p}$.
Mamine F., Montaigne E., Boutonnet J.-P., 2016. Perception de la qualité des produits laitiers et comportement du consommateur algérien. Econ. Rural. Agric. Aliment. Territ., 355: 49-65, doi: 10.4000/economierurale.4994

Nedjraoui D., 2001. Profil fourrager : Algérie (éds Neumann C.G. et al.). FAO, Rome, Italie, $30 \mathrm{p}$.

Ngom Y., Duteurtre G., Dia D., 2019. La régulation du marché du lait en poudre au Sénégal : compromis entre acteurs et logiques d'actions, Rev. Afr. Sci. Soc. Santé Publique, 1 (2): 116-138

Nguyen M.-H., Duteurtre G., Moustier P., 2017.What shapes the governance of the dairy value chain in Vietnam? Insights from Ba-Vì milkshed (Hanoi). World Food Policy, 3 (2-1): 57-81, doi: 10.18278/wfp.3.2.4.1.4

OCDE, 2005. Les échanges et l'ajustement structurel : les enjeux de la mondialisation. Les éditions de l'OCDE, Paris, France, 368 p.

ONIL, 2012. Les missions de l'ONIL entre régulation et appui au développement de la production laitière. $3^{\mathrm{e}}$ Forum sur la filière laitière, Souk Ahras, Algérie, 13-14 Juin 2012

Sari D., 2001. La crise algérienne économique et sociale : Diagnostic et perspectives. Eléments de stratégie. Publisud, Paris, France, 134 p.

Soukehal A., 2013. La sécurité alimentaire : quels programmes pour réduire la dépendance en céréales et lait ? Colloque, forum des chefs d'entreprises. Alger. FCE, 14 Avril 2013

\section{Summary}

Mamine F., Fares M., Duteurtre G., Madani T. Regulation of the dairy sector in Algeria between food security and development of local production: Review

Food security is at the heart of current debates on agricultural development policies. One major issue relates to balancing support to local production and opening up to international trade in order to meet the rapid demand growth. This question arises in an unusual way in the Algerian dairy sector, where state intervention concerns all links in the commodity chain from production to consumption. However, despite these particularly costly measures, the increase in the collection of local milk remains limited. The State also intervenes through mechanisms of administered prices and quotas, which encourage the import of milk powder in order to ensure the supply of low-price dairy products. This compromise maintains the country's dependence on international markets and calls into question its ability to develop a genuine strategy for food sovereignty.

Keywords: milk, dried milk, agricultural policies, food security, imports, Algeria

\section{Resumen}

Mamine F., Fares M., Duteurtre G., Madani T. Regulación del sector lechero en Argelia entre seguridad alimenticia y desarrollo de una producción local: síntesis

La seguridad alimenticia se encuentra al centro de los debates actuales relacionados con las políticas de desarrollo agrícola. Se trata principalmente de arbitrar entre el apoyo a la producción local y la apertura al comercio internacional para responder al rápido crecimiento de la demanda. Esta pregunta se presenta de forma original en el sector lechero argelino, donde la intervención del Estado concierne al conjunto de eslabones de la filial de la producción al consumo. Sin embargo, a pesar de estas medidas particularmente onerosas, el progreso de la colecta de leche local permanece limitado. El Estado interviene también mediante mecanismos de precios regulados y de cuotas, que favorecen la importación del polvo de leche con el fin de asegurar la oferta de productos lecheros a bajo precio. Este compromiso lleva al mantenimiento de la dependencia del país con respecto a los mercados internacionales y cuestiona su capacidad a elaborar una verdadera estrategia de soberanía.

Palabras clave: leche, leche en polvo, política agrícola, seguridad alimentaria, importaciones, Argelia 
